\title{
OSMOLARIDADE E TAXA DE DILUIÇÃO NA ATIVAÇÃO DO SÊMEN CRIOPRESERVADO DE PROCHILODUS LINEATUS
}

\author{
OSMOLARITY OF ACTIVATOR AND DILUTION RATE IN THEACTIVATION \\ OF CRYOPRESERVED SEMEN OF CURIMBA (PROCHILODUS LINEATUS)
}

Felizardo, V.O. ${ }^{1 A}$, Murgas, L.D.S. ${ }^{1 B}$, Navarro, R.D. ${ }^{1}$, Gonçalves, A.C.S. ${ }^{1 *}$ e Paulino, M.S. ${ }^{1}$

${ }^{1}$ Universidade Federal de Lavras. Lavras, Minas Gerais. Brasil. Aviviofbio@yahoo.com.br; BIsmurgas@ufla.br; *antonycarl2003@yahoo.com.br

\author{
PalaVRas chave adicionais \\ Motilidade espermática. Peixe.
}

\section{RESUMO}

Objetivou-se avaliar a influência de ativadores com diferentes osmolaridades e taxa de diluição na ativação de sêmen criopreservado de curimba (Prochilodus lineatus). Foram utilizados quatro reprodutores machos capturados na Estação de Piscicultura da CEMIG, Itutinga, MG. As amostras de sêmen foram diluídas em duas soluções (DMSO + lactose e metanol + lactose) na proporção de 1:4 e congeladas. Foram avaliadas as taxas (\%) e duração (s) da motilidade espermática dos tratamentos. Na ativação foram utilizados ativadores contendo as respectivas osmolaridades: 30, 99, 183 e 293 mOsm nas taxas de diluição de 1:2, 1:4, 1:6, 1:8 sêmen: ativador. A taxa de diluição 1:2 e 1:8 foram estatisticamente significativas para duração da motilidade para o sêmen criopreservado com DMSO. Para a diluição 1:2, o sêmen ativado com o ativador contendo $30 \mathrm{mOsm}$, apresentou duração de motilidade maior em relação ao sêmen ativado com ativadores com 183 e 293 mOsm. No entanto, para a utilização do ativador com $30 \mathrm{mOsm}$, se obteve maior duração da motilidade na diluição de 1:2 em relação a diluição de 1:4. Na taxa de diluição 1:6, quando o sêmen foi ativado com o ativador de $30 \mathrm{mOsm}$, a taxa de motilidade foi superior à apresentada pelo sêmen que foi ativado com $293 \mathrm{mOsm}$. Na taxa de diluição de 1:8, o uso de ativador contendo $99 \mathrm{mOsm}$ apresentou maior duração de motilidade em relação ao uso do ativador com 293 mOsm. Ativadores contendo altas osmolaridades podem proporcionar menores taxas e duração da motilidade em sêmen de curimba (Prochilodus lineatus) crio-

Recibido: 24-3-09. Aceptado: 12-5-10.

\author{
AdDitionAL KEYWORDS \\ Sperm motility. Fish.
}

preservado com os crioprotetores metanol ou DMSO.

\section{SUMMARY}

The objective of this work was to evaluate the influence of activators with different osmolarities and dilution rate in the activation of cryopreserved semen of curimba (Prochilodus lineatus). We used four breeding males captured in the Fishfarming Station of CEMIG, Itutinga, MG. Semen samples were diluted in two solutions (DMSO + methanol and lactose + lactose) at 1:4 ratio and frozen. The rates (\%) and duration (s) of sperm motility for treatments were assessed. Activators with respective osmolarities: 30, 99, 183 and 293 mOsm at dilution rates of 1:2, 1:4, 1:6, 1:8 (semen: activator) were used. The dilution rates of $1: 2$ and $1: 8$ influenced duration of motility in semen cryopreserved with DMSO. For the 1:2 dilution, the semen activated with the activator containing 30 mOsm, presented greater motility duration than semen activated with activators with 183 and 293 mOsm. However, for the activator with $30 \mathrm{mOsm}$, it was obtained longer motility duration at a dilution of $1: 2$ compared to $1: 4$ dilution. At 1:6 dilution rate, when the semen was activated with the activator $30 \mathrm{mOsm}$, the motility rate was higher than that provided by the semen that was activated with $293 \mathrm{mOsm}$. At the dilution rate of $1: 8$, the use of activator containing $99 \mathrm{mOsm}$ showed longer motility duration in relation to use of the activator with $293 \mathrm{mOsm}$. Activators containing high 
osmolarities can provide lower rates and duration of motility in semen of curimba (Prochilodus lineatus) cryopreserved with cryoprotectants methanol or DMSO.

\section{INTRODUÇÃO}

A capacidade técnica para preservação de gametas e embriões de peixes e de invertebrados aquáticos tem se expandido rapidamente nos últimos anos, impulsionada primariamente pela indústria aqüícola (Carolsfeld et al., 2003).

A criopreservação é uma técnica importante no controle reprodutivo de muitas espécies e sua aplicação em peixes está em amplo desenvolvimento. Envolve procedimentos que permitem o armazenamento de espermatozóides em nitrogênio líquido, mantendo-se a viabilidade dos gametas por tempo indefinido (Felizardo, 2008).

A criopreservação do sêmen tem sido testada para várias espécies como, por exemplo, o dourado Salminus maxillosus (Carolsfeld et al., 2003); a piracanjuba Brycon orbignyanus (Carolsfeld et al., 2003; Maria et al., 2006; Felizardo, 2008); o curimbatá Prochilodus lineatus (Felizardo et al., 2010; Murgas et al., 2007) e a tilápianilótica Oreochromis niloticus (Amorim, 2002).

No processo de criopreservação, a incorporação de soluções diluidoras é fundamental para proporcionar meio osmótico e nutricionalmente ideal aos espermatozóides durante a aplicação da biotecnologia. O BTS (Beltsville Thawing Solution) é um diluidor desenvolvido e preconizado para conservação do sêmen suíno. Entretanto, vários autores têm utilizado com sucesso esse diluidor em pesquisas com peixes de piracema, como piracanjuba (Maria et al., 2006; Felizardo, 2008), curimba (Murgas et al., 2007) e pirapitinga, Brycon nattereri (Oliveira et al., 2007).

Juntamente com o diluidor, é necessário o uso de um crioprotetor para que haja uma proteção do espermatozóide durante o congelamento e o descongelamento (Squires et al., 1999). Estes crioprotetores devem possuir como propriedades uma baixa toxicidade para as células e alta solubilidade em água. Os crioprotetores podem ser classificados como intracelulares ou permeáveis e extracelulares ou impermeáveis.

Entre os crioprotetores intracelulares mais utilizados podem ser citados dimetilsulfóxido (DMSO), glicerol, metanol e etilenoglicol. A gema de ovo e o leite em pó desnatado são os crioprotetores extracelulares mais comuns (Carolsfeld e Harvey, 1999).

A motilidade espermática é um dos principais parâmetros a serem considerados na análise da qualidade do sêmen de peixes (Godinho, 2000). Para tanto, deve-se levar em conta que a motilidade espermática é influenciada por inúmeros fatores como temperatura, estado nutricional, estado sanitário, condições de análise, solução ativadora e a proporção ativador:sêmen.

Em baixas proporções de ativador/ sêmen, somente alguns espermatozóides são ativados, enquanto outros têm sua ativação tardia. Este fenômeno deve-se à diluição insuficiente ou à mistura inadequada da solução ativadora com o sêmen (Chereguini et al., 1999).

Entre as soluções ativadoras da motilidade espermática, merecem destaque a água destilada, soluções salinas de bicarbonato de sódio, $\mathrm{NaCl}$ e $\mathrm{KCl}$ em diferentes concentrações, dependendo da espécie (Ravinder et al., 1997).

Miliorini et al. (2004) utilizaram $\mathrm{NaCl}(30$ e $60 \mathrm{mM}$ ) e $\mathrm{NaHCO}_{3}$ (30 e $60 \mathrm{mM}$ ) como soluções ativadoras do sêmen de $P$. lineatus resfriado. Silveira (2000) observou que a solução de $\mathrm{NaHCO}_{3} 1 \%$ favoreceu satisfatoriamente a taxa de motilidade do sêmen de matrinxã (Brycon cephalus), porém, os ovócitos, durante a fertilização, se aglutinaram no fundo do recipiente e a taxa de eclosão foi nula.

Dentre as espécies que tem se destacado em estudos de criopreservação pode- 


\section{ATIVADORES ETAXA DE DILUIÇÃO NA ATIVAÇÃO DO SÊMEN DE CURIMBA}

mos citar a curimba, que é uma espécie nativa, que necessita migrar com destino à cabeceira do rio durante o período de piracema, para realizar a reprodução. É uma das espécies de peixes de água doce com maior significado na piscicultura comercial (Cerqueira e Fernandes, 2002), sendo muito apreciada na culinária dos estados da região nordeste do Brasil (Maia et al., 1999).

Um dos principais aspectos que estimulam o desenvolvimento da biotecnologia que auxiliam na procriação da curimba é o fato de que suas larvas são muito bem utilizadas como alimento de espécies carnívoras na fase larval, além de servir como espécie-modelo no desenvolvimento de pesquisas reprodutivas, dadas sua elevada prolificidade e facilidade de manejo.

Nesse sentido objetivou-se verificar a influência de ativadores com diferentes osmolaridades e taxas de diluição na ativação de sêmen criopreservado de curimba.

\section{MATERIAISE MÉTODOS}

O experimento foi realizado na Estação de Piscicultura da Companhia Energética de Minas Gerais - CEMIG, em Itutinga, durante o período de piracema nos meses de novembro de 2006 a janeiro de 2007 . Foram selecionados quatro machos de curimba de peso médio de $1600 \mathrm{~g}$ que liberavam sêmen sob pequena compressão na parede celomática. Para a indução de liberação de gametas, foi ministrada uma dose única de $1 \mathrm{mg} / \mathrm{kg}$ intramuscular de extrato bruto de hipófise de carpa (EBHC) por kg de peso corporal.

Aproximadamente 196 horas graus após a aplicação do hormônio foi realizada a coleta do sêmen a seco, o volume de sêmen apresentado pelos machos foram de $2,43 \pm 0,94 \mathrm{ml}$. Logo após a coleta foi verificada a ausência de contaminantes como fezes, urina ou sangue. Uma alíquota de $10 \mu 1$ de sêmen foi coletada para se estimar a concentração espermática. Esta alíquota foi diluída em formol citrato na proporção de 1:100 (sêmen:formol citrato), antes da avaliação em microscópio, sendo para isto, utilizado câmara de New Bauer.

Foi avaliada a porcentagem de motilidade espermática do sêmen in natura em microscópio óptico e estimada a percentagem média de espermatozóides móveis. A duração da motilidade espermática foi estimada em segundos, desde a homogeneização com água destilada ( $1: 4$ sêmen:água destilada), até que somente $10 \%$ dos espermatozóides se encontrassem móveis. Somente amostras que apresentaram motilidade superior a $85 \%$ foram utilizadas para criopreservação.

Uma amostra de sêmen de cada animal foi diluída em solução crioprotetora contendo o diluidor BTS (Beltsville thawing solution) mais a combinação dos crioprotetores intracelulares metanol $8 \%$ e dimetilsulfóxido(DMSO) $8 \%$ e o extracelular lactose 5\%, na proporção de 1:4 (sêmen/ solução crioprotetora). O sêmen diluído foi envasado em palhetas de $0,5 \mathrm{ml}$, que foram vedadas com massa cirúrgicas e colocadas em botijão de vapor de nitrogênio líquido para resfriamento. Após 24 horas, as amostras foram transferidas para o botijão de armazenamento e mantidas até o descongelamento.

$\mathrm{O}$ descongelamento foi realizado dois anos após o congelamento no Laboratório de Fisiologia e Farmacologia do Departamento de Medicina Veterinária da Universidade Federal de Lavras. As palhetas foram imersas em banho-maria a $60^{\circ} \mathrm{C}$, durante oito segundos (Murgas et al., 2007; Felizardo, 2008), sendo em seguida, avaliado quanto à porcentagem (\%) e duração (s) da motilidade utilizando o mesmo processo que foi usado para avaliação do sêmen in natura.

Amostras de $2 \mu l$ de sêmen foram ativadas utilizando quatro diferentes soluções ativadoras: água destilada; $\mathrm{NaCl}$ $0,3 \% ; \mathrm{NaCl} 0,6 \%$ e $\mathrm{NaCl} 0,9 \%$. Foram testados quatros diferentes proporções de sêmen:ativador: 1:2, 1:4, 1:6, 1:8. Vale ressaltar que o sêmen criopreservado já estava diluído na proporção de 1:4 
sêmen:solução crioprotetora.

Este experimento foi conduzido, em um delineamento inteiramente casualizado, com os tratamentos em esquema fatorial de $4 \times 4$ x 2, sendo 4 concentrações de ativadores $(30,99,183,293 \mathrm{mM}), 4$ taxas de diluição (1:2, $1: 4,1: 6,1: 8$ sêmen:ativador) e 2 crioprotetores (DMSO e metanol), com 4 repetições, sendo cada macho como unidade experimental. Os dados foram submetidos a análises de variância, utilizando-se o pacote computacional SAS (1999). As variáveis taxa e duração da motilidade espermática foram comparadas pelo teste de Duncan a $5 \%$ de probabilidade.

\section{RESULTADOSEDISCUSSÃO}

As osmolaridades dos respectivos ativadores estão apresentadas nas tabelas. Os valores médios da taxa de motilidade (\%) do sêmen criopreservado com o crioprotetor DMSO, em função dos tratamentos estão apresentados na tabela $\mathbf{I}$.

No presente experimento a taxa de diluição de 1:2, 1:4 e 1:6 sêmen: ativador para o sêmen criopreservado com o crioprotetor DMSO não apresentaram diferença $(\mathrm{p}>0,05)$

Tabela I. Valores médios da taxa de motilidade (\%) do sêmen criopreservado com o crioprotetor DMSO, em função dos tratamentos estudados. (Average rate of motility (\%) of sperm cryopreserved with DMSO cryoprotector according to the treatments studied).

\begin{tabular}{|c|c|c|c|c|c|}
\hline \multirow[t]{2}{*}{ TD } & \multicolumn{4}{|c|}{ Concentração ativadores $\mathrm{mM}$} & \multirow[t]{2}{*}{$S$} \\
\hline & 30 & 99 & 183 & 293 & \\
\hline $1: 2$ & 50,0 & 51,3 & 35,0 & 26,3 & ns \\
\hline $1: 4$ & 48,8 & 55,0 & 32,5 & 25,0 & ns \\
\hline $1: 6$ & 50,0 & 48,8 & 37,5 & 18,8 & ns \\
\hline $1: 8$ & $46,3^{\mathrm{ab}}$ & $58,8^{a}$ & $33,8^{a b}$ & $10,0^{\mathrm{b}}$ & $p<0,05$ \\
\hline$S$ & $\mathrm{~ns}$ & ns & ns & ns & \\
\hline
\end{tabular}

TD: Taxa de diluição; S: Significância.

${ }^{a b}$ Letras distintas na mesma linha indicam diferença significativa $(p<0,05)$ pelo teste de Duncan. entre as osmolaridades testadas. No entanto, na diluição de 1:8 sêmen: ativador, o ativação com solução ativadora contendo 99 mOsm apresentou maior $(p<0,05)$ motilidade em relação ao sêmen ativado com ativador com 293 mOsm de osmolaridade.

Não foi observada diferença significativa $(p>0,05)$ na taxa de motilidade entre as taxas de diluição testadas na ativação do sêmen criopreservado com o crioprotetor DMSO.

Shimoda et al. (2007) testando os efeitos da osmolaridade sobre a motilidade espermática da piabanha (Brycon insignis), utilizaram nove concentrações de $\mathrm{NaCl}$ variando de 0 a $1,6 \%$. Os autores verificaram que osmolaridade de até $274 \mathrm{mOsm}$ ativou praticamente todos os espermatozóides, mas com 342 mOsm, apenas parte dos espermatozóides foram ativados e quando a concentração aumentou para 410 mOsm ou mais, não ocorreu ativação espermática.

Este fato pode ser explicado pelos ativadores com maiores concentrações de proporcionarem uma osmolaridade próxima à osmolaridade seminal, que para as espécies de água doce variam de 286 mOsm na piabanha (Shimoda et al., 2004) à $350 \mathrm{mOsm}$ em tilápia (Shimoda et al., 2007), e com isso não proporcionando meio adequado para ativação completa dos espermatozóides. Suquet et al. (1994) afirmam que altas pressões osmóticas inibem a motilidade espermática.

Carolsfeld et al. (2003) encontraram uma taxa de motilidade variando de 5 a $20 \%$ para o sêmen de curimba criopreservado com DMSO e ativado com $\mathrm{NaCl} 25 \mathrm{mmol}$, e quando utilizaram água destilada como ativador, obtiveram taxa de motilidade variando de 0 a $5 \%$.

Para ativação do sêmen in natura de piau-açu (Leporinus macrocephalus), Viveiros et al. (2003) verificou que osmolaridade de $50 \mathrm{mM}$ de $\mathrm{NaHCO}_{3}$ apresentou as maiores taxas de motilidade em relação as concentrações de 75 e 119 $\mathrm{mM}$. A diminuição da osmolaridade externa

Archivos de zootecnia vol. 60, núm. 232, p. 1258. 


\section{ATIVADORESE TAXA DE DILUIÇÃO NA ATIVAÇÃO DO SÊMEN DE CURIMBA}

imediatamente leva ao reajustamento da concentração iônica interna pelo processo osmorregulativo da membrana. A diminuição da concentração iônica atinge valores em que a atividade da ATPase é ótima e, conseqüentemente, a motilidade é em alta velocidade (Cosson, 2004).

Inoda e Morisawa, 1987 observou em espécies de água doce osmolaridade do sêmen ao redor de $250 \mathrm{mOsm}$. As baixas concentrações ( $<75 \mathrm{mOsm})$ e conseqüentemente, baixa osmolaridade ( $<150 \mathrm{mOsm})$ das soluções de $\mathrm{NaCl}$, promoveram as melhores taxas de motilidade espermática, classificando essas soluções como ativadoras.

Os valores médios para duração de motilidade do sêmen criopreservado com o crioprotetor DMSO, em função dos tratamentos estão apresentados na tabela II.

No presente experimento a taxa de diluição 1:2 e 1:8 sêmen:ativador foi estatisticamente significativa para duração da motilidade para o sêmen criopreservado com DMSO. Para a diluição 1:2 sêmen: ativador, o sêmen ativado com o ativador contendo $30 \mathrm{mOsm}$, apresentou uma duração de motilidade maior $(p<0,05)$ em relação ao

Tabela II. Valores médios de duração motilidade (s) do sêmen criopreservado com o crioprotetor DMSO, em função dos tratamentos estudados. (Average duration of motility (s) of sperm cryopreserved with DMSO cryoprotector according to the treatments studied).

\begin{tabular}{lccccc}
\hline TD & \multicolumn{6}{c}{ Concentração ativadores mM } & $\mathrm{S}$ \\
& 30 & 99 & 183 & 293 & \\
\hline $1: 2$ & $47,0^{\mathrm{a} A}$ & $24,0^{\mathrm{ab}}$ & $15,0^{\mathrm{b}}$ & $11,0^{\mathrm{b}}$ & $\mathrm{p}<0,05$ \\
$1: 4$ & $22,0^{\mathrm{AB}}$ & 26,0 & 18,0 & 6,0 & $\mathrm{~ns}$ \\
$1: 6$ & $17,0^{\mathrm{B}}$ & 25,0 & 24,0 & 5,0 & $\mathrm{~ns}$ \\
$1: 8$ & $19,0^{\mathrm{ABab}}$ & $31,0^{\mathrm{a}}$ & $25,0^{\mathrm{ab}}$ & $0,0^{\mathrm{b}}$ & $\mathrm{p}<0,05$ \\
$\mathrm{~S}$ & $\mathrm{p}<0,05$ & $\mathrm{~ns}$ & $\mathrm{~ns}$ & $\mathrm{~ns}$ & \\
\hline
\end{tabular}

TD: Taxa de diluição; S: Significância.

${ }^{A B a b}$ Letras distintas minúscula na mesma linha e maiúscula na coluna indicam diferença significativa $(p<0,05)$ pelo teste de Duncan. sêmen ativado com ativadores que possuíam osmolaridade de 183 e 293 mOsm. Já para a taxa de diluição de 1:8 sêmen: ativador, o sêmen ativado com ativador contendo 99 mOsm apresentou maior $(\mathrm{p}<0,05)$ duração de motilidade em relação ao sêmen ativado com ativador que possui osmolaridade de 293 mOsm.

As taxas de diluição de 1:4 e 1:6 sêmen:ativador não apresentaram diferença $(\mathrm{p}>0,05)$ para duração da motilidade para o sêmen criopreservado com DMSO, entre as osmolaridades testadas.

Estes resultados podem estar relacionados com as taxas de motilidade apresentadas quando se utilizou o ativador com maior osmolaridade, pois quanto menor a taxa de motilidade, menor será o tempo para que este sêmen atinja o tempo de parada da avaliação.

As taxas de diluição testadas na ativação do sêmen criopreservado não apresentaram diferença significativa $(\mathrm{p}>0,05)$ na duração da motilidade nas osmolaridades de 99, 183 e 293 mOsm. No entanto, para a utilização do ativador com osmolaridade de $30 \mathrm{mOsm}$, se obteve maior $(\mathrm{p}<0,05)$ duração da motilidade na diluição de 1:2 em relação a diluição de 1:4 sêmen:ativador.

Murgas et al. (2007) encontraram uma duração da motilidade média de 37 segundos para o sêmen de curimba criopreservado com DMSO e ativado com água destilada em uma taxa de diluição 1:4 sêmen: solução ativadora.

A duração da motilidade nula encontrada na diluição de 1:8 sêmen:ativador na osmolaridade de 293, se deve ao fato de que, neste trabalho a duração da motilidade era avaliada até que $10 \%$ dos espermatozóides estivessem móveis, por isso podemos observar uma taxa de motilidade de $10 \%$ neste tratamento, porém com tempo de duração nulo.

Os valores médios da taxa de motilidade (\%) do sêmen criopreservado com o crioprotetor metanol, em função dos trata- 
FELIZARDO, MURGAS, NAVARRO, GONÇALVES E PAULINO

mentos estão apresentados na tabela III.

Para o sêmen que foi criopreservado com crioprotetor metanol as taxas de diluição de 1:2, 1:4 e 1:8 sêmen: ativador não apresentaram diferenças significativa $(\mathrm{p}>0,05)$ para a taxa de motilidade espermática entre as osmolaridades testadas.

A taxa de diluição 1:6 apresentou diferença significativa $(\mathrm{p}<0,05)$, quando o sêmen foi ativado com o ativador que possui osmolaridade de $30 \mathrm{mOsm}$, a taxa de motilidade foi superior $(\mathrm{p}<0,05)$ à apresentada pelo sêmen que foi ativado utilizandose o ativador com 293 mOsm de osmolaridade. Ativadores contendo altas osmolaridades podem proporcionar menores taxas e duração da motilidade em sêmen de curimba criopreservado com os crioprotetores metanol ou DMSO.

Carolsfeld et al. (2003) encontraram taxa de motilidade variando de 80 a $100 \%$ para o sêmen de curimba criopreservado com metanol e leite em pó, ativado com $\mathrm{NaCl}$ $0,45 \%$.

As soluções ativadoras, usadas normalmente em sêmen de espécies nativas, se mostram espécie-específica. Mesmo quando os espermatozóides entram em contato com

Tabela III. Valores médios de motilidade (\%) do sêmen criopreservado com o crioprotetor metanol, em função dos tratamentos estudados. (Mean values of motility (\%) of sperm cryopreserved with methanol cryoprotector according to the treatments studied).

\begin{tabular}{lccccc}
\hline TD & \multicolumn{6}{c}{ Concentração } & \multicolumn{5}{c}{ ativadores mM } & S \\
& 30 & 99 & 183 & 293 & \\
\hline $1: 2$ & 45,0 & 28,0 & 28,0 & 25,0 & ns \\
$1: 4$ & 48,0 & 38,0 & 25,0 & 19,0 & ns \\
$1: 6$ & $60,0^{\text {a }}$ & $43,0^{\text {ab }}$ & $30,0^{\text {ab }}$ & $15,0^{\text {b }}$ & p $<0,05$ \\
$1: 8$ & 38,0 & 43,0 & 20,0 & 13,0 & ns \\
s & ns & ns & ns & ns & \\
& & & & &
\end{tabular}

TD: Taxa de diluição; S: Significância.

${ }^{a b}$ Letras distintas na mesma linha indicam diferença significativa $(p<0,05)$ pelo teste de Duncan. meios de baixa osmolaridade, ocorrem respostas diferentes entre variadas espécies e até mesmo entre o sêmen fresco e criopreservado da mesma espécie (Marques, 2001).

Shimoda et al. (2007) verificaram para o sêmen in natura de piabanha que a ativação utilizando diferentes concentrações de $\mathrm{NaCl}$ com osmolaridade variando de 0 a 274 mOsm proporcionaram taxas de motilidade semelhantes.

Os valores médios da duração da motilidade do sêmen criopreservado com o crioprotetor metanol, em função dos tratamentos estão apresentados na tabela IV.

No presente estudo as taxas de diluição 1:2, 1:4 e 1:6 sêmen: ativador utilizando o crioprotetor metanol, não apresentaram diferenças $(\mathrm{p}>0,05)$ no tempo de duração da motilidade espermática, entre as osmolaridades testadas. A ativação do sêmen na taxa de diluição 1:8 com a osmolaridade de 99 mOsm apresentou maior tempo de duração da motilidade em relação ao sêmen ativado com ativador de osmolaridade de 293 mOsm.

Não foi observada diferença significativa $(p>0,05)$ na duração da motilidade entre as taxas de diluição testadas na ativação do

$\overline{\text { Tabela IV. Valores médios de duração de }}$ motilidade (s) do sêmen criopreservado com o crioprotetor metanol, em função dos tratamentos estudados. (Average duration of motility (s) of sperm cryopreserved with methanol cryoprotector according to the treatments studied).

\begin{tabular}{lccccc}
\hline \multirow{6}{*}{ TD } & \multicolumn{6}{c}{ Concentração } & \multicolumn{5}{c}{ ativadores mM } & S \\
& 30 & 99 & 183 & 293 & \\
\hline $1: 2$ & 30,7 & 39,0 & 38,0 & 24,0 & ns \\
$1: 4$ & 33,0 & 38,0 & 25,0 & 19,0 & ns \\
$1: 6$ & 38,0 & 42,0 & 27,0 & 21,0 & ns \\
$1: 8$ & $40,0^{\text {ab }}$ & $45,0^{\text {a }}$ & $30,0^{\text {ab }}$ & $17,0^{\mathrm{b}}$ & $\mathrm{p}<0,05$ \\
$\mathrm{~S}$ & $\mathrm{~ns}$ & $\mathrm{~ns}$ & $\mathrm{~ns}$ & $\mathrm{~ns}$ &
\end{tabular}

TD: Taxa de diluição; S: Significância.

${ }^{a b}$ Letras distintas na mesma linha indicam diferença significativa $(p<0,05)$ pelo teste de Duncan.

Archivos de zootecnia vol. 60, núm. 232, p. 1260. 


\section{ATIVADORESE TAXA DE DILUIÇÃO NA ATIVAÇÃO DO SÊMEN DE CURIMBA}

sêmen criopreservado com o crioprotetor metanol.

\section{CONCLUSÃO}

Pode se observar através deste trabalho, que ativadores contendo altas osmolaridades podem proporcionar menores taxas e duração da motilidade em sêmen de curimba (Prochilodus lineatus) criopreservado com

\section{BIBLIOGRAFIA}

Amorim, V.M.C. 2002. Criopreservação de sêmen de tilápia-nilótica (Oreochromis niloticus), variedade chitralada. Dissertação (Mestrado em Zoologia). Pontifícia Universidade Católica de Minas Gerais. Belo Horizonte. 64 pp.

Carolsfeld, J. e Harvey, B. 1999. Conservação de recursos genéticos de peixes: teoria e prática. Curso de Treinamento Brasileiro. World Fisheries Trust. Victoria. $41 \mathrm{pp}$.

Carolsfeld, J., Godinho, H.P., Zaniboni Filho, E.B. and Harvey, J. 2003. Cryopreservation of sperm in Brazilian migratory fish conservation. J. Fish Biol., 63: 472-489.

Cerqueira, C.C. and Fernandes, M.N. 2002. Gill tissue recovery after copper exposure and blad parameter responses in the tropical fish, Prochilodus scrofa. Ecotox. Environ. Safe., 52: 83-91.

Chereguini, O., De la Banda, I.G., Rasines, I. and Fernandez, A. 1999. Artificial fertilization in turbot, Scopothalmus maximus, L.: different methods and determination of the optimal spermegg ratio. Aquac. Res., 30: 319-324.

Cosson, J. 2004. The ionic and osmotic factors controlling motility of fish spermatozoa Aquacult. Int., 12: 69-85.

Felizardo, V.O. 2008. Manejo reprodutivo da piracanjuba (Brycon orbignyanus): congelamento de sêmen e taxas de fertilidade. Dissertação (Mestrado em Ciências Veterinárias). Universidade Federal de Lavras. Lavras, MG. 84 pp.

Felizardo, V.O., Mello, R.A., Murgas, L.D.S., Andrade, E.S., Drumond, M.M. and Rosa, P.V 2010. Effect of cryopreservant combinations on the motility and morphology of curimba (Prochilodus lineatus) sperm. Anim. Reprod. os crioprotetores metanol ou DMSO.

\section{AGRADECIMENTOS}

À Companhia Energética de Minas Gerais CEMIG, pela fundamental colaboração na realização deste e de outros inúmeros trabalhos ligados à piscicultura, primando conosco pela preservação da fauna de Piracema e do ecossistema aquático.

Sci., 122: 259-263.

Godinho, H.P. 2000. Criopreservação de sêmen de peixes. Informe Agropecuário, 21: 6-20.

Inoda, T. and Morisawa, M. 1987. Effect of osmolality on the initiation of sperm motility in Xenopus laevis. Comp. Biochem. Phys., 88: 539-545.

Maia, E.L., Oliveira, C.C.S., Santiago, A.P., Cunha, F.E.A., Holanda, F.C.A.F. e Sousa, J.A. 1999. Composição química e classes de lipídios em peixe de água doce Curimatã comum, Prochilodus cearensis. Ciênc. Tecn. Aliment., 19: 433-437.

Maria, N.A., Viveiros, A.T.M., Freitas, R.T.F. and Oliveira, A.V. 2006. Extenders and cryoprotectants for cooling and freezing of piracanjuba (Brycon orbignyanus) semen, an endangered Brazilian teleost fish. Aquaculture, 260: 298306.

Marques, S. 2001. Preservação em curto prazo do sêmen de teleósteos neotropicais de água doce. Dissertação (Mestrado em Zoologia). Pontifícia Universidade Católica de Minas Gerais. Belo Horizonte. 83 pp.

Miliorini, A.B., Murgas, L.D.S., Viveiros, A.T.M. and Pereira, G.M. 2004. The effects of cryoprotectants and activators on sperm motility of curimba (Prochilodus lineatus). In: International Congress on Animal Reproduction, 15. Anais... Porto Seguro. pp. 523.

Murgas, L.D.S., Miliorini, A.B., Freitas, R.T.F. e Pereira, G.J.M. 2007. Criopreservação do sêmen de curimba (Prochilodus lineatus) mediante adição de diferentes diluidores, ativadores e crioprotetores. Rev. Bras. Zootecn., 36: 526531.

Oliveira, A.V., Viveiros, A.T.M., Maria, A.N., Freitas, 


\section{FELIZARDO, MURGAS, NAVARRO, GONÇALVESEPAULINO}

R.T.F. e Izaú, Z.A. 2007. Sucesso do resfriamento e congelamento de sêmen de pirapitinga Brycon nattereri. Arq. Bras. Med. Vet. Zootec., 59: $1509-1515$

Ravinder, K., Nasarunddin, K., Majumdar, K.C. and Shivaji, S. 1997. Computerized analysis of motility patterns and parameters of spermatozoa of carp following short-term storage of semen. $J$. Fish. Biol., 50: 1309-1328.

SAS. 1999. SAS Procedures guide for computers. $6^{a}$ ed. Cary, NC. 3: 373 pp.

Shimoda, E., Andrade, D.R. e Vidal Junior, M.V. 2004. Análise química do plasma seminal da piabanha Brycon insignis. Em: I Congresso da Sociedade Brasileira de Aquicultura e Biologia Aquática. Vitória-ES. Anais... pp. 194.

Shimoda, E., Andrade, D.R., Vidal Junior, M.V., Yasui, G.S., Silva, J.F.S. e Godinho, H.P. 2007. Efeito da osmolaridade sobre a motilidade espermática na piabanha Brycon insignis. Ceres, 54: 430:433.
Silveira, A.N. 2000. Caracterização espermática, preservação criogênica do sêmen e fertilidade do matrinxã (Brycon cefálios). Dissertação (Mestrado em Ciências Biológicas). Universidade Estadual Paulista. Botucatu, SP. 45 pp. Squires, E.L., Pickett, B.W., Graham, J.K., Vanderwall, D.K., McCue, P.M. and Bruemmer, J.E. 1999. Principles of cryopreservation. In: Cooled and frozen stallion semen. Fort Collins: Animal Reproduction and Biotechnology Laboratory. Colorado State University. pp. 9.

Suquet, M., Billard, R., Cosson, J., Dorange, G., Chauvaud, L., Mugnier, C. and Fauve, L.C. 1994. Sperm features in turbot (Scophthalmus maximus): a comparison with other freshwater and marine fish species. Aquat. Living Resour., 7: 283-294

Viveiros, A.T.M, Jatzkowski, A. and Komen, J. 2003. Effects of oxytocin on semen release response in African catfish (Clarias gariepinus). Therm. Eng., 59: 1905-1917.

Archivos de zootecnia vol. 60, núm. 232, p. 1262. 